\title{
INTRODUCCIÓN AL ESTUDIO DE SEDIMENTOS FLUVIALES DE LLANURA
}

Dra. SUÁREZ, Paola A. ${ }^{1}$; Dr. ORFEO, Oscar ${ }^{2}$; Dra. VEGA, Marisol ${ }^{3}$

\section{RESUMEN}

Con la finalidad de conservar los ambientes fluviales en sus condiciones prístinas, diversas variables deben ser analizadas. Los sedimentos son unas de las variables más importantes dado que influye potencialmente la morfología del paisaje, la química de las aguas y la dinámica biológica de los cursos de agua. El presente trabajo presenta una introducción de fácil interpretación sobre las características de mayor relevancia ambiental de los sedimentos, sus interacciones y su dinámica en áreas de llanura, de tal modo que los científicos que trabajen en ambientes fluviales de llanura puedan tener una primera aproximación para una correcta interpretación ambiental del rol de los sedimentos. Las características aquí mencionadas incluyen clasificación granulométrica, transporte y mineralogía.

En las últimas décadas aumentaron las investigaciones sobre estudios orientados a la evaluación de ambientes fluviales con el objeto de mejorar el conocimiento de su estructura y dinámica, para así poder trabajar en mantenerlos lo más cercano posible a sus condiciones prístinas, mediante una correcta gestión de los recursos involucrados.En el presente trabajo se realiza una breve introducción a los fundamentos que rigen el estudio de los sedimentos en ambientes fluviales de llanura, una de las variablesa tener en cuenta, junto a las características físicas y químicas de las aguas, así como de los suelos, cuando se pretende realizar un estudio del estado y evolución de un ambiente. Una correcta interpretación de los ambientes de llanura resulta indispensable para los científicos que trabajan en la región, dado que la Llanura Chacopampeana y la región Mesopotámica, son áreas caracterizadas por la baja pendiente.

\section{Sistemas fluviales y las llanuras}

Existen diversas propiedades por las cuales los sistemas fluviales pueden ser clasificados, las dos más relevantes para este trabajo son: 
(1) Schumm (2003) propuso una división dentro de cada río en tres zonas, teniendo en cuenta un sistema fluvial ideal:

- Zona 1: "Área de aporte de sedimentos". Aquí se encuentran los tributarios, constituidos por sistemas de vectores dendríticos arborescentes que irrigan la cuenca. Es la zona de producción primaria de la cuenca, donde se acumula flujo líquido/ sólido y se caracteriza por el desarrollo progresivo de la energía cinética y mecánica, que se manifiesta por una vigorosa erosión de fondo e incipiente erosión de márgenes (Campoblanco Díaz y Gomero Torres, 2000).

-Zona 2: "Área de transferencia", donde predomina el transporte de sedimentos y puede existir un equilibrio entre la entrada y la salida de estos. Recibe de la zona $1 \mathrm{ma}^{-}$ terial tanto sólido como líquido, presenta un aumento del caudal y de la energía cinética, con erosión predominante tanto de fondo como de las márgenes.

-Zona 3: "Área de depósito", considerada de gran importancia por su implicación ambiental. Campoblanco Díaz y Gomero Torres (2000) identifican esta zona con la presencia de humedales. Aquí la erosión de fondo disminuye, al tiempo que aumenta en las márgenes. Tiene lugar la lenta selección de la carga sólida transportada, dejando atrás los clastos de mayor tamaño, mientras que los de mediano y pequeño tamaño se transportan hasta las áreas de deposición.

(2) En una revisión realizada por Makaske (2001), se presenta un resumen de varios autores sobre la clasificación de los sistemas fluviales de acuerdo a la sinuosidad, S, y al número de canales activos. La Tabla 1 muestra una de esas clasificaciones, realizada por Rust en 1978, que resulta ser la más sintética y explicativa.

\begin{tabular}{|c|c|c|}
\hline $\begin{array}{c}\text { Baja sinuosidad } \\
(S<1,5)\end{array}$ & Unicanalizado & Multicanalizado \\
\hline $\begin{array}{c}\text { Alta sinuosidad } \\
(S>1,5)\end{array}$ & Meandroso & Antrelazado \\
\hline
\end{tabular}

Tabla 1. Clasificación de los sistemas fluviales según Rust (1978).

Los denominados ríos de baja sinuosidad son resultado de estructuras geológicas como fallas y pliegues, mientras que los ríos de alta sinuosidad son más frecuentes en la naturaleza y se encuentran generalmente en zonas de llanura o baja pendiente, donde desarrollan amplias áreas inundables con canales que suelen tener una migración lateral importante.

Se consideran "áreas de llanuras" a aquellos territorios que presentan una pendiente del terreno de 1 a $40 \mathrm{~cm} / \mathrm{km}$. Paoli y Giacosa (1983) señalan que el 35\% de la superficie continental de Argentina está conformada geomorfológicamente por llanuras, en su mayoría inundables, donde los sistemas fluviales no se comportan dentro de los parámetros establecidos para ríos típicos. Es por esta razón que Fertonani y Prendes (1983) consideran que estas unidades hidrológicas no se desarrollan bajo el concepto tradicional de cuencas y denominan a los sistemas fluviales de llanura como "Sistemas Hidrológicos No Típicos" (SHNT). Los SHNT se caracterizan por 
presentar una superficie de drenaje poco definida, con componentes de disipación lineales escasamente organizados y jerarquizados que impiden la identificación precisa de los límites del sistema. En ellos la estructura de disipación se caracteriza por la amortiguación ante una entrada energética (precipitación), acumulando agua e incluso inundando gran parte del área si fuera necesario, en lugar de generar escurrimiento como lo harían los Sistemas Hidrológicos Típicos"(SHT).

Debido a las particulares características de funcionamiento de las llanuras, se generan limitaciones a las posibilidades de manejo y utilización de estos espacios. El efecto de "encharcamiento" que ocurre en las áreas de bajas pendientes, a causa del retraso en el escurrimiento superficial del agua procedente de las precipitaciones, es uno de los efectos más importante (Paoli y Giacosa, 1983). Este retraso en el escurrimiento aumenta el almacenamiento, la infiltración y la evaporación, generando que los coeficientes de escorrentía sean claramente más bajos que en las cuencas con pendiente marcada. Durante los períodos húmedos se produce un ascenso importante del nivel freático, lo que disminuye la aireación, favorece la saturación total del terreno y el afloramiento del agua en superficie. En estas áreas de llanura, los ríos desarrollan baja energía para transportar sedimentos, por lo que el mecanismo dominante de movilización de materiales suele ser en suspensión (Blasi, 1981).

En los sistemas fluviales típicos, los cursos de agua ubicados en el área de de- pósito o desembocadura son receptores de la resultante de los eventos meteorológicos y de los procesos que transforman el ambiente en los sectores más elevados de sus cuencas (salinización, erosión, prácticas culturales, acción del fuego, etc). De acuerdo a Neiff (1986), esta premisa requiere salvedades para los paisajes de llanura subtropical donde los cursos de agua salen de su cauce durante las fases de grandes lluvias, generando un palco de inundación de recurrencia y duración altamente variable, que influyen sobre la estructura y dinámica de los ecosistemas comprometidos, incluso aquellos situados en las partes más elevadas de los gradientes topográficos. Así, los cursos de agua no son sólo efecto de procesos ocurridos en sus áreas de aporte.

\section{Los sedimentos y su importancia en} los ambientes fluviales

La dinámica fluvial tiene un papel muy importante en la irrigación de los ecosistemas terrestres y en la conexión de las cuencas con la atmósfera, los mares y los océanos. La diversidad de paisajes que podemos observar a lo largo de los continentes depende en gran medida de la presencia o ausencia del agua, así como de la erosión, el transporte y la sedimentación de materiales, generada por acción de su movimiento.

A lo largo de millones de años, los ríos han transportado gran parte de los sedimentos que afloran en las orogenias y que forman parte del sustrato litológico de las placas continentales. Así, los ríos no sólo forman parte importante del ciclo hidrológico sino también del ciclo de la formación 
de las rocas, la orogenia y la denudación de los continentes (Sabater y Elosegi, 2009).

Los sistemas fluviales son los responsables de la transferencia de materiales desde los continentes hacia las cuencas oceánicas. De acuerdo a Nanson y Gibling (2004), las cuencas de los ríos ocupan cerca del 69\% de la superficie de la corteza terrestre continental y transportan alrededor de $19 \mathrm{bi}^{-}$ llones de toneladas de materiales por año, de los cuales el 20\% aproximadamente se realiza en solución. Esta dinámica de transporte desedimentos y solutos se encuentra determinada porun conjunto de complejas variablesque interactúan, tales como la litología, el clima y el relieve. Lacomposición química del agua de los ríos es por consiguiente un reflejo delas complejas relaciones naturales e interdependientes entre las precipitaciones, la meteorización delos minerales, el ciclo de la vegetación, y de la evolución de la historia de su agua (Depetris y Pasquini, 2007).

Los sedimentos son materiales no consolidados que se generan durante los procesos de meteorización que sufren las rocas al estar expuestos a las condiciones ambientales; por lo tanto, las propiedades de los sedimentos se encuentran en función de la geología de la cuenca. Algunos procesos de meteorización fragmentan físicamente la roca sin modificar su composición original, mientras que otros la modifican químicamente dando lugar a nuevos minerales y a sustancias muy solubles en agua. El material resultante de los procesos de meteorización puede ser transportado por el agua, el viento o el hielo glacial, hasta los lugares donde sedimentan cuando la energía del medio de transporte disminuye (Tarbuck y Lutgens, 2005).

Una de las clasificaciones que admiten los sedimentos es la distinción entre cohesivos y no cohesivos, y aunque no existe una delimitación clara entre ellos, se considera no cohesivos a los sedimentos con tamaño de grano $>60 \mu \mathrm{m}$, mientras que aquellos $<2 \mu \mathrm{m}$ (arcillas) son generalmente cohesivos. Aunque los granos de tamaño más fino son considerados los más adherentes, los limos $(2 \mu \mathrm{m}-60 \mu \mathrm{m})$ presentan propiedades aglutinantes debido a la presencia de las arcillas, por lo que en la práctica, limos y arcillas son considerados materiales cohesivos (Huang et al. 2006).

Las partículas finas que forman parte de los materiales cohesivos presentan uniones fuertes entre ellas debido a sus cargas iónicas superficiales, por lo que en estas circunstancias, son las fuerzas entre las partículas, y no la fuerza gravitacional, las que dominan el comportamiento de los sedimentos (Hayter, 1983; Berlamont et al., 1993; Huang et al.,2006). Los materiales cohesivos además forman parte de los denominados lodos, presentes en muchos ambientes sedimentarios fluviales y de estuarios, y los cuales además se encuentran conformados por aguas (salina o salobre), materia orgánica (detritos de animales y plantas, bacterias, etc) y pequeñas cantidades de arenas y limos (Berlamont et al., 1993).

Entre las clasificaciones de sedimentos existen diversas escalas que analizan el intervalo de tamaños sin tener en cuenta su comportamiento, algunas aplican criterios 


\begin{tabular}{|c|c|c|c|c|c|}
\hline Grados & & $\mathrm{mm}$ & Fracción & Micrón & $\begin{array}{l}\text { Tamiz } \\
\text { ASTM }\end{array}$ \\
\hline \multirow{4}{*}{ Grava } & Bloque & 256 & & & \\
\hline & Guijón & 64 & & & \\
\hline & Guijarro & 4 & & & 5 \\
\hline & Gránulo & 2 & & & 10 \\
\hline \multirow{5}{*}{ Arenas } & $\begin{array}{l}\text { Muy } \\
\text { gruesa }\end{array}$ & 1 & & & 18 \\
\hline & Gruesa & 0,5 & $1 / 2$ & 500 & 35 \\
\hline & Mediana & 0,25 & $1 / 4$ & 250 & 60 \\
\hline & Fina & 0,125 & $1 / 8$ & 125 & 120 \\
\hline & Muy Fina & 0,0625 & $1 / 16$ & 62,5 & 230 \\
\hline \multirow{4}{*}{ Limos } & Grueso & 0,031 & $1 / 32$ & 31 & \multirow{4}{*}{$P$} \\
\hline & Mediano & 0,0156 & $1 / 64$ & 15,6 & \\
\hline & Fino & 0,0078 & $1 / 128$ & 7,8 & \\
\hline & Muy Fino & 0,039 & $1 / 256$ & 3,9 & \\
\hline \multirow{6}{*}{ Arcilla } & & 0,0020 & $1 / 512$ & 2,0 & \multirow{6}{*}{$\begin{array}{l}\mathrm{P} \\
\mathrm{E} \\
\mathrm{T} \\
\mathrm{A}\end{array}$} \\
\hline & & 0,00098 & $1 / 1024$ & 0,98 & \\
\hline & & 0,00049 & $1 / 2048$ & 0,49 & \\
\hline & & 0,00024 & $1 / 4096$ & 0,24 & \\
\hline & & 0,00012 & $1 / 8192$ & 0,12 & \\
\hline & & 0,00006 & $1 / 16384$ & 0,06 & \\
\hline
\end{tabular}

Tabla 2. Comparación de escalas y denominación de los tamaños de grano (Adaptada de Perillo, 2003).

geométricos y otros logarítmicos (Tabla 2). La escala diseñada por Udden (1894) es una escala geométrica de razón 2, que separa a los grupos en "grados". Posteriormente esta nomenclatura fue modificada por Wenworth en 1922, quien estableció nuevos límites para cada grado y es la escala más usada en la actualidad. La escala propuestas por Udden y modificada por Wenworth (1992).

Interacciones de los sedimentos con la columna de agua

Las propiedades del agua de los ríos, así como las de los sedimentos, se encuentran influenciadas por la composición geológica de la cuenca fluvial. De los materiales acarreados por los ríos, gran parte se incorporan a causa de la erosión que éstos producen a su paso. Según Pouey (1998), la magnitud del proceso erosivo está regulada no sólo por las características de los suelos superficiales, sino también por el flujo, la pendiente y la situación climática.

Conjuntamente, de los fenómenos de meteorización y desintegración de rocas en ambientes subacuáticos, una parte de la roca se solubiliza químicamente y otra parte pasa a suspensión en estado coloidal; los elementos en solución en el medio acuoso son precipitados químicamente en función 
de modificaciones en el $\mathrm{pH}$, Eh y la temperatura (Sabater y Elosegi, 2009).

Muchos nutrientes y contaminantes (como los metales pesados y los plaguicidas) se fijan a las partículas finas mediante fenómenos de adsorción y, en menor medida, de absorción, aumentando la densidad y velocidad de precipitación de los sedimentos (Domínguez et al., 2004). De esta forma, los sedimentos influyen sobre la calidad del agua de los ríos, participando como reservorios, medio de transporte y medio de dispersión de esos materiales ( $\mathrm{Zhu}$ et al., 2008; Horowitz, 1991). Los procesos de adsorción pueden producirse en diferente grado y ello queda determinado por la existencia de propiedades físico-químicas tanto de los suelos y/o sedimentos como de los productos químicos. Los parámetros más importantes que condicionan los procesos de unión son: el contenido de carbono orgánico, el contenido de arcilla, la humedad, el $\mathrm{pH}$, la capacidad de intercambio catiónico y la temperatura, entre otros (Porta et al., 2003). Las numerosas reacciones químicas y biológicas que ocurren en los cuerpos de agua fluviales pueden modificar las condiciones de $\mathrm{pH}$ y potencial redox del agua, alterando los equilibrios y liberando los elementos contenidos en los sedimentos, los cuales pasan a la columna agua.

Si bien la contaminación de los sedimentos es uno de los sucesos más importantes debido al deterioro de la calidad del recurso hídrico, alteraciones en la dinámica de transporte sedimentario también ocasionan graves consecuencias ambientales. De acuerdo a la FAO (2002) y Huang et al.
(2006), los sedimentos pueden considerarse ellos mismos como potenciales contaminantes de los recursos hídricos tanto desde el punto de vista tanto físico como químico. El aumento de la turbidez del agua y la sedimentación como factores físicos de contaminación no pueden ser despreciados, el primero por limitar la entrada de luz solar al cuerpo de agua y el segundo por la colmatación que genera en las cuencas.

\section{Dinámica de transporte de los sedi- mentos \\ Los fenómenos de transporte de sedi- mentos son de especial interés en la hidro-} logía fluvial para resolver tanto cuestiones ambientales como de ingeniería (McLaren y Bowles, 1984; Habibi, 1994; Ackermann y Schubert, 2007; Friese et al., 2007; Symader et al., 2007; entre muchos otros).

E1 flujo del agua y del aire define el transporte de los sedimentos; sin embargo, la forma en que se produce, así como las condiciones que definen el inicio y fin del mismo, dependen de las propiedades de los sedimentos (Perillo, 2003). De acuerdo al ciclo de movilización de materiales, el agente pierde capacidad de transporte a medida que se incrementa la distancia desde la fuente de procedencia, sobreviniendo así el proceso de sedimentación (Spalletti, 1986), el cual está influenciado por el tipo y cantidad de carga que transporta. Esta pérdida de energía por parte del agente de transporte genera una distribución gradada en sentido horizontal y el material más fino alcanza zonas muy alejadas a las áreas de nacientes. 
La flora y la fauna influyen claramente en el transporte de los sedimentos, incluso en aquellos ambientes que a priori se supone que no afectan, como desiertos, altas cumbres, regiones polares y fondos abisales. Las plantas, por su parte, en especial sus raíces, disminuyen el transporte de los sedimentos al actuar como barreras que disminuyen la velocidad de las corrientes. Los animales influyen en el transporte de sedimentos de dos formas diferentes: (1) aumentando la disponibilidad de sedimentos para ser transportados a causa de las bioturbaciones que producen y (2) al generar sustancias aglutinantes que convierten a los materiales inconsolidados en cohesivos, aumentando el tamaño de granos por las aglomeraciones y la energía de los agentes de transporte que se necesita para moverlos (Elosegi et al., 2009).

Existen diversos mecanismos por los que los materiales pueden ser transportados a lo largo de los ríos. Siguiendo la clasificación de Spalletti (1986) tenemos:

- Tracción: desplazamiento de los individuos por el sustrato o lecho de la corriente. En el rodamiento (o rólido) la movilización es por rotación alrededor del eje mayor del grano, mientras que en el deslizamiento (o arrastre)el clasto se desplaza según un plano que es comúnmente el que contiene a sus ejes mayor e intermedio.

- Saltación: movimiento a saltos, provocados por fuerzas de alzamiento hidráulico que tienen su origen generalmente en el choque entre los granos.

- Suspensión: es el transporte de los individuos en el seno de la corriente.
- Flotación: es el movimiento de material detrítico en la superficie del medio líquido.

- Solución: es el desplazamiento de componentes en estado iónico en un agente líquido.

El desplazamiento de las partículas clásticas por tracción y suspensión depende de la intensidad del flujo, de su densidad y viscosidad, así como de la granulometría, forma y densidad de los componentes. Por su parte, la dinámica de transporte de sedimentos en suspensión requiere especial atención al establecer zonas de acumulación y resuspensión de los contaminantes, así como aumentar el tiempo de residencia de los mismos en el sistema. Por norma general, las partículas más redondeadas, de menor tamaño y menor densidad son transportadas con mayor facilidad (Spa1letti, 1986).

La sedimentación es el proceso por el cual el material transportado se deposita. Este proceso puede llevarse a cabo tanto en partículas gruesas como finas debido a reducción local o general de la capacidad de transporte, donde la velocidad y la turbulencia se reduzcan (Tarbuck y Lutgens, 2005).

Los sedimentos de tamaño más fino tienden a unirse formando unidades de mayor tamaño, con diferentes posibilidades de transporte y velocidad de sedimentación que las partículas individuales. Este proceso depende del tipo y la concentración de sedimento, de la concentración iónica en la columna de agua (principalmente la salinidad) y de las condiciones del flujo (Mehta 
et al. 1989; Berlamont et al., 1993; Huang et al. 2006). El contenido de algunos metales (hierro, aluminio y calcio) y de materia orgánica también influye en los procesos de atracción entre los sedimentos. Estos agregados tienen la particularidad de crecer cuando colisionan con otras partículas o agregados, pero también pueden romperse por efecto de la turbulencia.

En los ambientes fluviales, una vez que los sedimentos son depositados comienzan a formar parte del lecho superficial del río, induciendo cambios cualitativos en dichos estratos. Horowitz (1991) señala que los sedimentos ubicados a una cierta profundidad pueden utilizarse como un registro histórico de los cambios que han tenido lugar en la cuenca, ya que no se encuentran influenciados por las recientes alteraciones físicas ni químicas de superficie.

Así mismo, en las áreas de bajas pendientes es frecuente que, durante los períodos húmedos, el cauce principal de los ríos se desborde provocando inundaciones donde el material transportado en suspensión se deposita en el territorio lateral, generando una acreción vertical (Allen, 1964; Douglas, 1977; Ritter, 1978; Ottesenet al., 1989; Brown, 1996). Se denomina "llanura aluvial" o "llanura de inundación" al territorio que ocupa el río en estos períodos. Algunos autores (Ottesenet al., 1989; Nanson y Croke, 1992; Bölvikenet al., 1996; Sweenen y Van der Sluys, 1998; Sweenen y Van der Sluys, 2002; Adánezet al., 2009; Suárez et al., 2016) sugieren que, mediante el estudio geoquímico de los sedimentos de la llanura aluvial, es posible determinar niveles bases, así como detectar cambios ocasionados en los mismos por la actividad antrópica.

\section{La mineralogía de arcillas}

Por medio del análisis mineralógico de sedimentos es posible identificar su composición y las condiciones físico-químicas presentes en el momento de su génesis (Bertolino, 2006). Estos análisis pueden llevarse a cabo sobre cualquiera de las fracciones granulométricas presentes en los depósitos sedimentarios. Sin embargo, de las rocas y los minerales que constituyen la litósfera terrestre, los minerales de tipo arcillosos son los de mayor importancia ambiental debido a las interacciones que facilitan.

En mineralogía, el término arcilla (gr. Argilos=blanco) es utilizado para hacer referencia a un conjunto de minerales de tamaño de partícula $<4 \mu \mathrm{m}$, los cuales pertenecen predominantemente a la subclase de los filosilicatos (Porta et al., 1994). Se trata de aluminosilicatos hidratados con estructura en hojas, espaciamientos y contenidos en los espacios interlaminares que son propios para cada mineral. Pueden presentar sustituciones del aluminio por $\mathrm{Fe}$ o $\mathrm{Mg}$ y álcalis, así mismo pueden estar compuestos por un solo mineral arcilloso o estar mezclados con otros minerales como feldespatos, cuarzo, carbonatos y micas (Klein y Hurlbut, 1993).

Las arcillas juegan un papel importante en las características que presentan tanto los suelos como los sedimentos, debido a su plasticidad, a su capacidad de formar soluciones coloidales, de retener agua, de intercambiar iones $\mathrm{y}$, sobre todo, de formar agregados. Particularmente, las arcillas son 
productos de reacciones de alteración de las rocas terrestres por meteorización y es por ello que son muy estudiadas; pueden proceder de minerales preexistentes en el material originario, por herencia, transformación o neoformación debido a los procesos de meteorización (Porta et al., 1999). Además, debido a su estructura cristalina y a la carga neta superficial negativa (que les permite adsorber cationes y materia or- gánica) son intercambiadores muy eficaces de iones, que impactan en la composición química de los ríos (Huerta Díaz, 1998). En ambientes lacustres, las asociaciones de diversos argilominerales reflejan la litología, el tipo de suelos y el clima del área de aporte (Chamley, 1989). Al identificar el grupo de arcillas dominante en un depósito sedimentario, es posible predecir su comportamiento e impacto en el ambiente estudiado.

\section{BIBLIOGRAFÍA}

- Ackermann, F. y Schubert, B. (2007) Trace Metals as Indicators for the Dynamics of (Suspended) ParticulateMatter in the Tidal Reach of the River Elbe. En: Sediment Dynamics and Pollutant Mobility in Rivers. An Interdisciplinary Approach. Springer, EnvironmentalScience and Engineering. 430pp.

- Allen, J. R. L. (1964) Studies in fluviatile sedimentation. Six cyclothems from the Lower Old Red Sandstone, Anglo-Welsh. 166-198pp.

- Berlamont, J.; Ockenden, M.; Toorman, E.; Winterwero, J. (1993) The characterization of cohesive sediments properties. Coastal Engineering, 21: 105-128.

- Bertolino, S. R. A. (2006) Arcillas y ambientes sedimentarios. Curso de actualización. IV Congreso Latinoamericano de sedimentología. XI Reunión argentina de sedimentología. S. C. Bariloche, Argentina.

- Blasi, A. (1981) Relación tamaño de grano-selección en sedimentos actuales. Asoc. Min. Petr.
Sed. Rev. 12 (1-2): 1-10pp.

- Bölviken, B.; Bogen, J.; Demetriades, A.; De Vos, W.; Ebbing, J.; Hindel, R.; Langedal, M.; Locutura, J.; O'Connor, P.; Ottesen, R. T.; Pulkkinen, E.; Salminem, R.; Schermann, O.; Swennen, R.; Van der Sluys, J.; Volden, T. (1996) Regional geochemical mapping of Western Europe towards the year 2000. Journal of Geochemical Explotation, 56: 141-166pp.

- Brown, A. G. (1996) Floodplain Palaeoenvironments. En: Anderson, M. G., Walling, D. E y Bates, P. D. (ed.), Floodplain Processes. John Wiley and Sons, Chichester, U. K., 94-138pp.

- Chamley, H. (1989) Clay Sedimentology. Springer-Verlag (Ed.), Berlin,623pp.

- Campoblanco Díaz, H.; Gomero Torres, J. (2000) Importancia de los ríos en el entorno ambiental. Rev. Inst. investig. Fac. minas metal cienc. geogr, vol.3, no.5: 57-63pp.

- Depetris, P.J.; Pasquini, A.I. (2007) The Geochemistry of the Paraná River: An Overview. In The 
Middle Paraná River: Limnology of a Subtropical Wetland: 6: 143-174pp.

- Domínguez-Chicas, A.; Kretzschmar, T.; Núñez-Sánchez, F. (2004) Velocidades de sedimentación en aguas pluviales de Cd. Juárez, Chihuahua, México. Revista Mexicana de Ciencias Geológicas. Vol. 21 (3), 412-420pp.

- Douglas, I. (1977) Humid Landforms. Australian National University Press, Canberra, 288 pp.

- Elosegi, A.; Butturini, A.; Armengol, J. (2009) El caudal circulante. En: Elosegi, A.y Sabater, S. (Eds.): Conceptos y técnicas de ecología fluvial: 4: 51-69pp.

- FAO (2002) Relaciones tierra-agua en cuencas hidrográficas rurales. Actas del taller electrónico organizado por la Dirección de Fomento de Tierras y Aguas de la FAO. Boletín de Tierras y aguas de la FAO 9.89pp.

- Fertonani M, Prendes H (1983) Hidrología en áreas de llanura: aspectos conceptuales, teóricos y metodológicos, En: Hidrología de Grandes Llanuras Vol. I: Argentina, UNESCO, 119-156pp.

- Friese, K.; Schwartz, R.; Krüger, Frank. (2007) Transport and Storage of River Sediment and Associated Trace Metals into Floodplains of the Elbe. En: Sediment Dynamics and Pollutant Mobility in Rivers. An Interdisciplinary Approach. Springer, EnvironmentalScience and Engineering. 430pp.

- Habibi, M. (1994) Sediment transport estimation methods in river systems. $\mathrm{PhD}$ Thesis.

- http://ro.uow.edu.au/theses/1263/ (ultimo acceso 10/07/ 2013).

- Hayter, E.J. (1983) "Prediction of Cohesive Sediment Movement in Estuarial Waters," Ph.D. Thesis, the University of Florida at Gainesville.

- Horowitz, A.J. (1991) A primer on trace metalsediment chemistry. 2da Edic. U.S. Geological
Survey Water-Supply. 136pp.

- Huang,J.; Hilldale, R. C.; Greimann, B.P. (2006)

Cohesive Sediment Transport, en: Reclamation Managing Water in the West (Ed.): Erosion and Sedimentation Manual: 4: 1-54pp.

- Huerta Díaz, M. A. (1998)

- http://iio.ens.uabc.mx/Curso\%20Internet\%20 Miguel\%20Angel/2_Arcillas/Arcillas.htm (última visita 10/09/2013)

- Klein, C.; HurlbutJr, C. S. (1993) Manual of Mineralogy. 21st ed. $681 \mathrm{pp}$.

- Makaske, B. (2001) Anastomosing rivers: a review of their classification, origin and sedimentary products. Earth-Science Reviews 53: 149196pp.

- McLaren, P.; Bowles, D. (1984) The effects of sediment transport on grain-size distributions. Journal od Sedimentary Petrology, Vol. 5 (4): 457-470pp.

- Mehta, A.J.; Hayter, E.J.; Parker, W.R.; Krone, R.B.; Teeter, A.M. (1989) Cohesive Sediment Transport. En: Process Description, Journal of Hydraulic Engineering, vol. 115, no. 8: 10761093pp.

- Nanson, G.C.; Croke, J.C. (1992) A genetic classification of floodplains. Geomorphology, 4: 459-486pp.

- Nanson, G.C.; Gibling, M.R. (2004) Rivers and Alluvial Fans. Major article for Encyclopedia of Sediments and Sedimentary Rocks, (Ed.) G.Middleton, Kluwer, 568-583pp.

- Neiff, J.J. (1986) Sinópsis ecológica y estado actual del Chaco oriental. Revista Ambiente Subtropical 5-35pp.

Ottesen, R. T.; Bogen, J.; Bölviken, B.; Volden, T. (1989) Overbank sediment: a representative sample medium for regional geochemical mapping. Journal of Geochemical Exploration, 32: 
257-277pp.

- Paoli, C.; Giacosa, R. (1983) Necesidades de investigaciones hidrológicas en áreas de llanuras. En: Hidrología de Grandes Llanuras Vol. I: Argentina, UNESCO, 119-156pp.

- Perillo, G. M.E. (2003) Dinámica del transporte de sedimentos. Asociaciónn Argentina de Sedimentología, 201pp.

- Porta, J.; Lopez-Acevedo, M.; Roquero De Laburu, C. (1999) Edafología para la agricultura y el medio ambiente. 2a Edición. Mundi Prensa (Ed.), España. 849pp.

- Porta Casanellas, J.; López-Acevedo Reguerín, M. y Roquero de Laburu, C. (2003) Edafología para la agricultura y el medio ambiente. $3^{\text {a }}$ Edición. Ed. Mundi-Prensa. Madrid. 929pp.

- Pouey, N. (1998) Erosión Hídrica en Cursos de Llanura sobre Lechos Cohesivos, UNR Editora, Rosario, Argentina. 164pp.

- Ritter, D.F. (1978) Process Geomorphology. W.C. Brown, Iowa, 603 pp.

- Rust, B.R. (1978) A classification of alluvial channel systems. En: Miall, A.D. (Ed.), Fluvial Sedimentology. Canadian Society of Petroleum Geologists Memoir 5, Calgary, pp. 187-198pp.

- Sabater, S.; Elosegi, A. (2009) Presentación: importancia de los ríos. En: Elosegi, A. y Sabater, S. (Eds.): Conceptos y técnicas de ecología fluvial Fundación BBVA. España.

- Schumm, S. A. (2003) The fluvial system. Ed. The Blackburn Press. New Jersey, USA. 338pp.

- Spalletti, L.A. (1986) Nociones sobre transporte y depositación de sedimentos clásticos. Rev. Fac. Cienc. Nat. y Museo, UNLP, Serie Téc. yDid. No 13. 102pp.

- Symader W.; Bierl, R.; Kurtenbach, A.; Krein, A.
(2007) The relevance of River Botton Sediments for the transport of Cohesive Particles and Attached contaminants. En: Sediment Dynamics and Pollutant Mobility in Rivers. An Interdisciplinary Approach. Springer, EnvironmentalScience and Engineering. 430pp.

- Suárez, P.A.; Vega, M.; Pardo, R.; Orfeo, O.; García-Cuesta, J.L.; Ronco, A. (2016) Hydrochemical and Sedimentological Dynamics in a Subtropical Plain River: Assessment by Multivariate Statistical Analysis. Environmental Earth Sciences, 75: 1004.

- Swennen, R; Van der Sluys, J. (1998) Zn, Pb, Cu and As distribution patterns in overbank and medium-order stream sediment samples: their use in exploration and environmental geochemistry. Journal of Geochemical Exploration, 65: 27-45pp.

- Swennen, R; Van der Sluys, J. (2002) Anthropogenic impacto $\mathrm{n}$ sediment composition and geochemistry in vertical overbank profile of river alluvium from Belgium ans Luxembourg. Journal of Geochemical Exploration, 75: 93-105pp.

- Tarbuck, E.J.; Lutgens, F.K. (2005) Ciencias de la Tierra. Una introducción a la geología física. 8va. Edic. 736pp.

- Udden, J. A. (1894) Erosion, Transportation and sedimentation performed by the Atmosphere. Journal of Geology, 2: 318-331pp.

- Wentworth, C. K. (1922) A scale of grade and class terms for clastic sediments. Journal of Geology, V. 30: 377-392pp.

- Zhu, Y.; Lu, J.Y.; Liao, H. Z.; Wang, J. S.; Fan, B. L.; Yao S. M. (2008) Research on cohesive sediment erosion by flow: An overview. Science in China Series E: Technological Sciences. Springer. Vol. 51. No. 11. 